\title{
e-interview
}

columns

\section{Lynn DeLisi}

Lynn DeLisi is Professor of Psychiatry, New York University, NY; Associate Director of The Center for Advanced Brain Imaging and The Nathan S. Kline Institute for Psychiatric Research; Secretary/Treasurer of The International Society of Psychiatric Genetics and co-editor-in-chief, Schizophrenia Research. She trained at St Elizabeth's Hospital, Washington and The National Institute of Mental Health (NIMH), Bethesda, Maryland.

\section{If you were not a psychiatrist, what} would you do?

I can think of a lot of things that I would like to do other than be a psychiatrist. First, would probably have studied anthropology or archaeology - obtained a PhD in one of these and developed a career researching human evolution. A second choice might have been to be an international photographic journalist, recording and writing about unusual human interest happenings world-wide.

What has been the greatest impact of your profession on you personally? This is a difficult question to answer and the answer is certainly not all positive. I strive for perfection and to be the very best at what I am doing. Thus, my whole career, I have worked long and hard hours sacrificing much of my personal life. My quest has been to find a biological cause for schizophrenia. So with that in mind, I still have a long way to go if this is to be achieved in my lifetime. The politics of science and peer competition, along with the constant struggle to maintain funding support, have taken a toll on my personal well-being. However, on the positive side, I have felt pride in the occasional evidence of progress in the brain imaging and genetic studies I have pursued.

\section{Do you feel stigmatised by your profession?}

lam not sure what this question means. However, when my views of the science are different from the mainstream view or what my most-accepted colleagues think as correct, I feel I struggle harder to retain funding support and recognition for my work. Since no one really knows the real truths about schizophrenia, respect and flexibility in thought are important among colleagues.

\section{What are your interests outside of} work?

I edit a journal - mostly 'after hours' and am constantly working when I am not travelling. I suppose exploring unknown places and observing how people live in other cultural and socioeconomic environments have always been exciting to me. I've also had an interest in clinical work in response

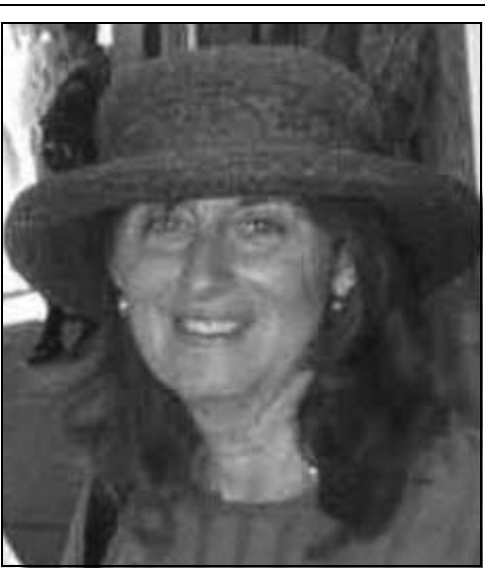

to disasters, i.e. I was part of a medical team that went to El Salvador after the 2001 earthquake and I helped out in NYC after the $9 / 11$ disaster.

What job gave you the most useful training experience?

was a researcher in the intramural programme at $\mathrm{NIMH}$ for 9 years. I probably would not have advanced as far as I have without that experience and am sorry I left. Pursuing research in an academic setting where clinical and other responsibilities prevail leaves less time to focus intensely on research, which is my passion.

Which book has influenced you most? Derek Bickerton wrote a book published in 1990 called Language and Species.

What research publication has had the greatest influence on your work?

Tim Crow's paper with John Done in the 1980s, drawing my attention to age of onset in pairs of siblings with schizophrenia. They emphasised the importance of heritable transmission of age of onset, rather than environmental transmission. That was one of a series of papers that convinced me that pursuing viral causes would not get me anywhere and I turned to genetics.

What part of your work gives you the most satisfaction?

Performing a statistical analysis of tediously collected data for several months and finding that something of importance has a $P$ value $<0.05$

\section{What do you least enjoy?}

Laboratory work - particularly when it involves mixing buffers and getting them just right, or going through a day or even months of experiments to find out that they didn't work.

What is the most promising opportunity facing the profession? I believe that genetic studies (maybe not in the way most people are pursuing them now, but using other methods) will eventually enable us to develop a whole new diagnostic system of serious psychiatric diseases. This will cross the boundaries of the conditions currently defined in the DSM and other clinical diagnostic criteria.

What single change would substantially improve quality of care? A pharmaceutical agent that is aimed at the brain developmental anomalies that occur in many of these illnesses before the obvious symptoms are recognised.

Do you think psychiatry is brainless or mindless?

Psychiatry has always been considered a 'soft' field in USA medical schools, except when the dean happens to be a psychiatrist. Most MDs consider psychiatrists experts on 'human behaviour'. They are of course wrong. Until we have clear biological causes for illnesses that are accepted to be 'psychiatric', this field will always stand apart. Note that Alzheimer's disease and Huntington's chorea used to be 'psychiatry', but are now 'neurological diseases' because they have underlying biology.

How would you entice more medical students into the profession?

By showing that we truly are a 'hard'science and not just 'talk' medicine.

How would you improve clinical psychiatric training?

Encourage more research time. Without research in this of all fields, clinical psychiatrists just flounder in how they provide help to patients.

How should the role of the American Psychiatric Association change? I recently terminated my membership of the American Psychiatric Association. This organisation does not serve the needs of academic salaried psychiatrists, nor clinical researchers in the field, and charges a huge annual fee requiring members to not only belong to the national association, but to a local branch, paying dues to both. Its annual meeting has become an international pharmaceutical company circus and social event, and no longer provides the high-quality academic medicine that I remember it having 20 years ago.

What is the future for psychotherapy in psychiatry training and practice? I believe not much except social support.

What single area of psychiatric research should be given priority? I believe that genetic strategies will lead us toward new treatments and thus should be given the most priority, but they need to be carefully discussed because there are many genetic strategies that will also lead us astray for years to come.

Dominic Fannon 